\title{
Resuscitation policies for extremely preterm newborns: finally moving beyond gestational age
}

\author{
Mark R. Mercurio $\mathbb{1}^{1,2} \cdot$ Brian S. Carter $\mathbb{1}^{3,4,5}$
}

Received: 18 August 2020 / Revised: 24 August 2020 / Accepted: 18 September 2020 / Published online: 2 October 2020

(c) Springer Nature America, Inc. 2020

Over a decade ago, Tyson et al. presented a strong, rational, and statistically robust argument for, in their words, "moving beyond gestational age" when assessing outcomes data for extremely low birthweight (ELBW) newborns [1]. Since that time, compelling arguments have been made by others for considering factors in addition to gestational age, but today guidelines based solely or primarily on estimated gestational age (eGA) prevail [2-5]. In this issue of the Journal, De Proost et al. highlight problems with this approach, prevalent in their national policy in the Netherlands. They aptly call for a change in their nation's policy, and movement away from gestational age-based guidelines in general [6].

The authors use illustrative vignettes to contrast the cases of a 23 3/7-week-female fetus at $600 \mathrm{~g}$ with a $241 /$ 7 -week male at 500 grams. They note that, despite a better chance of survival for the girl, her parents would not be given the option of resuscitation while the boy's parents would, according to current national guidelines in the Netherlands. Based on this apparent injustice, the threshold for resuscitation could be moved from 24 to 23 weeks. This, however, would only move the injustice, not rectify it-such that the same apparent unfairness could then be found when comparing options given to

Mark R. Mercurio

mark.mercurio@yale.edu

1 Department of Pediatrics, Yale University School of Medicine, New Haven, CT, USA

2 Program for Biomedical Ethics, Yale University School of Medicine, New Haven, CT, USA

3 Department of Pediatrics, University of Missouri-Kansas City School of Medicine, Kansas City, MO, USA

4 Department of Medical Humanities \& Bioethics, University of Missouri-Kansas City School of Medicine, Kansas City, MO, USA

5 Children's Mercy Bioethics Center, Kansas City, MO, USA parents of a larger 22-week girl to those of a smaller 23week boy.

Justice requires that equals be treated equally. If individuals are to be treated differently, a morally relevant difference should be identified between them. In creating guidelines for resuscitation of extremely preterm neonates, that difference has commonly been eGA or birthweight. Both miss the mark and do not represent morally relevant differences. Both eGA and birthweight have been used individually as tools to predict the likelihood of survival, or survival without significant impairment. At one point in time this might have been the best we could do, but this has not been the case for many years.

Current guidelines based on eGA use it as a proxy for a more reasonable and morally relevant difference among newborns when resuscitation decisions are made: prognosis. Prognosis entails the likelihood of the success of both resuscitation and neonatal intensive care. In the periviability context, one can argue that birth weight $<400 \mathrm{~g}$ and/or an eGA $<22$ completed weeks of pregnancy confer an especially poor prognosis $[7,8]$. No outcome data currently exist that suggest attempted resuscitation and intensive care for babies this small or young is morally compelled. However, we recognize that for certain borderline cases above these thresholds the prognosis, while worrisome or ambiguous, should allow parents a choice of whether to attempt resuscitation. Clinician judgment remains pertinent in gauging a response of the newborn to initial resuscitation, and initiation of critical care in the Neonatal Intensive Care Unit (NICU). Justice, writ large, is not contingent upon eGA or BW in so much as whether the possibility of benefit exists, and the commitment to weighing proportionate goods and harms lies with the continuum of care going forward in the NICU. This continuum is on the order of minutes to hours initially, and then settles out to days and weeks.

Even in consideration of justice as here applied, there will be some threshold below which parents are commonly not given the option of attempted neonatal resuscitation and intensive care. This is consistent with current professional 
guidelines and common practice and is not here disputed $[7,8]$. It is essential, however, that if parents are denied that option, there be a valid ethical justification. That justification is, or should be, determined again by a calculus of proportionate harm - that the likelihood of benefit for the baby is disproportionately small when measured against the likelihood of harm to the baby. And while resuscitation may not be provided, supportive comfort care for the baby and the family should be. Beyond these limits, it remains the responsibility of the neonatologist to regularly address such current and future harms with the parents. In this manner an individualized prognostic approach is modeled, and acceptable risks communicated by the parents. This may yield different treatment paths, some parents opting for continued life-extending treatments while others perhaps opting for a redirection towards comfort and palliative care.

We recognize that in some settings a valid justification might also be that the resources are not available, the resuscitation not feasible, or someone else has a greater claim on those resources [9]. Absent these, however, it comes down to predicted outcome of the baby, and for that one can and should do better than using eGA alone.

Tyson et al. demonstrated that eGA, birth weight, sex, plurality, and the receipt of antenatal steroids could be combined to predict survival and disability better than using gestational age alone and developed an on online tool to calculate such predictions. This year, Rysavy et al have once again shown that these factors combined are superior to any of them alone, including eGA, in predicting survival, and a new online calculator is available [10]. Hence, there is no clear ethical justification for institutional or national policies regarding resuscitation of ELBW newborns based on eGA alone.

Prognosis helps us to understand the likelihood of success. "Success" may be defined as survival to discharge, survival without profound neurodevelopmental impairment, or survival without any impairment. A thorough analysis of the relevance of impairment, and its social interpretation as disability, to resuscitation decisions is beyond the scope of this essay. But we would suggest that parents not be denied the option of resuscitation because of known or anticipated impairment or the child's feared disability. We suggest that success be defined as "survival" or as "survival without profound disability," as predicted by the NIH tool. The moral relevance of prognosis should be given voice by parents enjoined by their physician in a shared and deliberative manner such that the child's best interests are served. Some families may see harm in the frustrations of continued NICU care with all that comes with it, only to have a severely impaired child whose life will be fraught with disability; they might see striving despite these things as subjecting the child to an unfair/unjust life in the short or long-term.
Parents deserve transparency in how clinicians view and weigh varied outcomes. In speaking to anticipated impairment and potential disability, significant deference to the values and judgment of informed parents seems most appropriate, with rare exception. Regardless of whether profound disability is included, and thus how success is defined, multifactorial tools provide a better measure than eGA alone. While perhaps easier and more straightforward, using eGA too easily leads to the injustices described above. Identifying thresholds for when attempted resuscitation should be offered, and for when it should be obligatory, in terms of percent survival or success will be difficult but necessary, out of fairness to newborns and parents, and to optimize operations and patient care. What is made available to parents awaiting an imminent delivery should not change as physicians hand-off to one another over days or weeks. Agreed-upon guidelines should already be in place, and all involved in the care of a specific newborn should be onboard with the plan.

Prior to addressing any specific case, neonatology groups should meet to discuss relevant (including local) data, the need for moving beyond eGA, the relevant rights and other ethical issues at play, and pragmatic considerations, and then reach consensus on what their guidelines should be. Other important stakeholders, such as Obstetricians/Maternal-Fetal Medicine physicians and NICU nurses, should be invited to the evolution and implementation of the guidelines. This will be important for consistency in counseling and for optimizing outcomes. For example, if resuscitation becomes an option earlier than it would have under previous guidelines, antenatal steroid use should also be considered earlier. While consensus and consistency among the care team and in the guidelines are important, it should also be appreciated that whatever guidelines are established, some measure of flexibility may be appropriate to accommodate specifics of a given case not reflected in the multifactorial outcomes tool utilized.

DeProost et al make a good argument for changing the national policy in the Netherlands. Much of that reasoning, and a clearer understanding of how best to predict outcomes, compels a change in policy in many other countries as well, including the United States. The foundation of the argument essentially comes down to justice. It is a breach of justice to deny the option of resuscitation to one child yet offer it to another with a worse prognosis. Basing ELBW guidelines on eGA alone will inevitably lead to such injustice. At one point, perhaps, it was the best we could do. That is no longer the case, and it is time we moved beyond eGA alone as a resuscitation criterion.

Author contributions MRM co-developed the framework and arguments for the manuscript, wrote the first draft of the manuscript, and participated in revisions. BSC co-developed the framework and arguments for the manuscript, and participated in revisions of the manuscript. 


\section{Compliance with ethical standards}

Conflict of interest MRM receives royalty fees as an author of the entry on periviability for the online publication Up-To-Date. BSC declares no conflict of interest.

Publisher's note Springer Nature remains neutral with regard to jurisdictional claims in published maps and institutional affiliations.

\section{References}

1. Tyson JE, Parikh NA, Langer J, Green C, Higgins RD. National Institute of Child Health and Human Development Neonatal Research Network. Intensive care for extreme prematurity-moving beyond gestational age. N Engl J Med. 2008;358:1672-81. https://doi.org/10.1056/NEJMoa073059.

2. Wyckoff MH, Aziz K, Escobedo MB, Kapadia VS, Kattwinkel J, Perlman JM, et al. Part 13: Neonatal Resuscitation: American Heart Association guidelines update for cardiopulmonary resuscitation and emergency cardiovascular care (reprint). Pediatrics. 2015;136: s196-218. https://doi.org/10.1542/peds.2015-3373G.

3. Cummings J.American Academy of Pediatrics Committee on Fetus and Newborn Antenatal counseling regarding resuscitation and intensive care before 25 weeks of gestation. Pediatrics. 2015;136:588-95. https://doi.org/10.1542/peds.2015-2336.

4. Raju TN, Mercer BM, Burchfield DJ, Joseph GF. Periviable birth: executive summary of a Joint Workshop by the Eunice
Kennedy Shriver National Institute of Child Health and Human Development, Society for Maternal-Fetal Medicine, American Academy of Pediatrics, and American College of Obstetricians and Gynecologists. J Perinatol. 2014;34:333-42. https://doi.org/ 10.1038/jp.2014.70.

5. Nuffield Council on Bioethics. Critical care decisions in fetal and neonatal medicine: ethical issues. London, England: Nuffield Council on Bioethics; 2006.

6. De Proost L, Ismaili M'hamdi H, Verhagen AAEE, Reiss I, Geurtzen R, Verweij EJJ. On the limits of viability: toward an individualized prognosis-based approach. J Perinatol. 2020. https://doi.org/10.1038/s41372-020-00793-x.

7. Brumbaugh JE, Hansen NI, Bell EF, Sridhar A, Carlo WA, Hintz SR. for the National Institute of Child Health and Human Development Neonatal Research Network, et al. Outcomes of extremely preterm infants with birth weight less than $400 \mathrm{~g}$. JAMA Pediatr. 2019;173:434-45.

8. Patel RM, Rysavy MA, Bell EF, Tyson JE. Survival of infants born at periviable gestational ages. Clin Perinatol. 2017;44:287-303. https://doi.org/10.1016/j.clp.2017.01.009.

9. Mercurio MR, Cummings CL. Critical decision making in pediatrics: the I-P-O framework. J Perinatol. 2020. https://doi.org/ 10.1038/s41372-020-00841-6.

10. Rysavy MA, Horbar JD, Bell EF, Li L, Greenberg LT, Tyson JE. for the Eunice Kennedy Shriver National Institute of Child Health and Human Development Neonatal Research Network and Vermont Oxford Network, et al. Assessment of an Updated Neonatal Research Network Extremely Preterm Birth Outcome Model in the Vermont Oxford Network. JAMA Pediatr. 2020;174:e196294. https://doi.org/10.1001/jamapediatrics.2019.6294. 